\title{
The Druggable Antimalarial Target PfDXR: Overproduction Strategies and Kinetic Characterization
}

\author{
Jessica L Goble ${ }^{\mathrm{a}}$, Hailey Johnson ${ }^{\mathrm{a}}$, Jaco de Ridder ${ }^{\mathrm{b}}$, Linda L Stephens ${ }^{\mathrm{a}}$, Abraham Louw ${ }^{\mathrm{b}}$, Gregory L \\ Blatch $^{\mathrm{a}, \mathrm{c}}$ and Aileen Boshoff*,a
}

\begin{abstract}
${ }^{a}$ Biomedical Biotechnology Research Unit, Department of Biochemistry, Microbiology and Biotechnology, Rhodes University, Grahamstown 6140, South Africa; ${ }^{b}$ Department Biochemistry, University of Pretoria, Pretoria 0001, South Africa; ${ }^{c}$ School of Biomedical \& Health Sciences, Victoria University, Melbourne, Australia
\end{abstract}

\begin{abstract}
Plasmodium falciparum 1-deoxy-D-xylulose-5-phosphate reductoisomerase (PfDXR) is a key enzyme in the synthesis of isoprenoids in the malaria parasite, using a pathway that is absent in the human host. This enzyme is receiving attention as it has been validated as a promising drug target. However, an impediment to the characterisation of this enzyme has been the inability to obtain sufficient quantities of the enzyme in a soluble and functional form. The expression of PfDXR from the codon harmonised coding region, under conditions of strongly controlled transcription and induction, resulted in a yield of $2-4 \mathrm{mg} / \mathrm{L}$ of enzyme, which is 8 to 10 -fold higher than previously reported yields. The kinetic parameters $\mathrm{K}_{\mathrm{m}}, \mathrm{V}_{\max }$ and $\mathrm{k}_{\mathrm{cat}}$ were determined for PfDXR using an NADPH-dependent assay. Residues K295 and K297, unique to species of Plasmodium and located in the catalytic hatch region; and residues V114 and N115, essential for NADPH binding, were mutated to resemble those found in E. coli DXR. Interestingly, these mutations decreased the substrate affinity of PfDXR to values resembling that of E. coli DXR. PfDXR-K295N, K297S and PfDXR-V114A, N115G demonstrated a decreased ability to turnover substrate by 4-fold and 2-fold respectively in comparison to PfDXR. This study indicates a difference in the role of the catalytic hatch in capturing substrate by species of Plasmodium. The results of this study could contribute to the development of inhibitors of PfDXR.
\end{abstract}

Keywords: Plasmodium falciparum, DXR; anti-malarial, heterologous expression, molecular chaperones.

\section{INTRODUCTION}

Plasmodium falciparum 1-deoxy-D-xylulose-5phosphate reductoisomerase (PfDXR) is a vital enzyme in the 2-C-methyl-D-erythritol-4-phosphate (MEP) pathway for the production of isoprenoids in the malaria parasite. This pathway is different to that of the mevalonate pathway in the human host, making this enzyme an attractive anti-malarial drug target [1]. Recently PfDXR was genetically validated using a single cross-over strategy, and the enzyme was found to be essential for intraerythrocytic development of the parasite, further validating this enzyme as a chemotherapeutic target [2]. Malaria is a devastating parasitic disease caused by vector-transmission of five different species of Plasmodium protozoa, with P. falciparum being the most deadly strain [3].

The heterologous production of proteins in a bacterial expression system has been largely documented in the literature because of simplicity, rapid growth, and successful overproduction of the target protein [4]. However, a major limitation is the formation of insoluble non-functional clusters of protein known as inclusion bodies, which accumulate due to the attraction of exposed hydrophobic regions of mis-

\footnotetext{
*Address correspondence to this author at the Department of Biochemistry, Microbiology and Biotechnology, Rhodes University, PO Box 94, Grahamstown, 6140, South Africa; Tel: +27-46-603-8630; Fax: +27-46-622-3984; E-mail: a.boshoff@ru.ac.za
}

folded protein [5]. Another setback faced is degradation by the proteolytic machinery of the cell $[6,7]$.

The heterologous production of 1000 P. falciparum proteins in E. coli was carried out in a single study and 337 of these target proteins were expressed as insoluble aggregates [8]. The mRNA of $P$. falciparum is characterized by high $\mathrm{A} / \mathrm{T}$ content and an abundance of lysine and arginine repeats, which often leads to the early termination of translation resulting in the formation of truncated protein forms or insoluble inclusion bodies [9]. Amino acid codons used most frequently in P. falciparum are different to those used by other organisms [10]. Experimental strategies that have been adopted in order to overcome these obstacles include: rare codon tRNA supplementation [11], codon optimization [10, 12] and codon harmonization [13].

Rare codon tRNA supplementation involves coexpression of the target protein with a RIG plasmid that encodes transfer RNAs for arginine (R), isoleucine (I) and glycine $(G)$ [11]. This strategy was used successfully for the production of full-length $P$. falciparum heat shock protein 70 (PfHsp70) [14]. Codon optimization is an experimental strategy in which the coding region of a target protein is designed to include the most frequently used codons of the host cell [12]. However, the expression of many malaria proteins was not improved through codon optimization [15]. Codon harmonization is an alternative strategy that involves substituting codons with synonymous ones that have the same or similar usage frequencies in the expression host, while main- 
taining the integrity of the pause site profile of a native sequence [13]. Codon harmonization permits translation in the heterologous host in a similar way to the native host, and potentially enhances protein folding, unlike the product of an optimized gene [13].

The co-expression of molecular chaperones is an alternative method that has been used successfully to enhance the expression and solubility of target proteins. The molecular chaperones used can either be heterologous or homologous to the target protein. When overproduction of a target protein is induced in a host, the cell's endogenous chaperone system can become overloaded, thereby limiting the amount of soluble protein that can be produced [5]. The overproduction of molecular chaperones has been used successfully to enhance the yield of numerous target proteins [7, 16,17], however this technique has been found to be highly protein and chaperone specific [5].

In prokaryotes, the most physiologically important and ubiquitous chaperones can be divided into two groups: DnaK-DnaJ-GrpE and GroEL-GroES, which have distinct but co-operative functions [16]. DnaK (prokaryotic Hsp70 homologue) requires the co-chaperone DnaJ (prokaryotic Hsp40 homologue) and nucleotide exchange factor GrpE [18]. The mechanism of action relies on the binding and hydrolysis of ATP [18]. DnaK binds substrates via hydrophobic protein sequences of four to five residues flanked by basic residues [19]. GroEL (Hsp60 homologue) forms a threedimensional barrel structure containing a hydrophilic interior, with GroES (Hsp10) forming the lid [20]. Substrates bind GroEL via hydrophobic interactions on the interior rim of the hydrophilic chamber [21] and require subsequent binding of GroES and ATP to trigger the release of the protein into the cavity.

In 2002 the crystal structure of E. coli DXR (EcDXR) revealed the enzyme to be a homodimeric V-shaped molecule [22]. In the same year, the crystal structure of EcDXR in complex with NADPH was solved; and revealed the presence of a flexible loop that acted as a catalytic hatch that closed over the active site securing the substrate, and played an important role in the enzymatic reaction and substrate specificity [23]. Recently the crystal structure of PfDXR demonstrated an intrinsic flexibility of the enzyme to house inhibitors in the active site [24]. A model of PfDXR had been generated previously that showed structural similarity between E. coli and PfDXR, but the model was not publically available [25]. This presented an opportunity to produce a more vigorously validated PfDXR model and it was found that amino acid residues in the catalytic hatch were highly conserved amongst DXR homologues, with the exception of residues K295 and K297, that are unique to species of Plasmodium [26]. The conserved amino acid residues A35 and G36 of EcDXR are positioned to accommodate the 2' phosphate moiety of NADPH [22], and these are substituted for valine and asparagine respectively within species of Plasmodium.

In this study, we demonstrate the successful overproduction and purification of soluble active PfDXR using a codon harmonized coding region. The strategy also included the co-expression of molecular chaperones from both E. coli and $P$. falciparum. We show how codon harmonization, coupled to tight control of transcription, greatly improved heterologous protein production. The kinetic parameters of the purified PfDXR enzyme were also determined and compared to EcDXR. Residues unique to Plasmodium and present in the catalytic hatch and involved in the binding of NADPH to PfDXR were mutated by site-directed mutagenesis to resemble those found in EcDXR. A three-dimensional structure of monomeric PfDXR in complex with NADPH and an inhibitor (FR900098, an N-acetyl derivative of fosmidomycin) is shown in (Fig. 1) and it highlights the positions of these selected residues (V114, N115, K295 and K297). To our knowledge, these particular amino acid residues unique to PfDXR have not been mutated previously and the results could play a role in the rational design of novel DXR inhibitors.

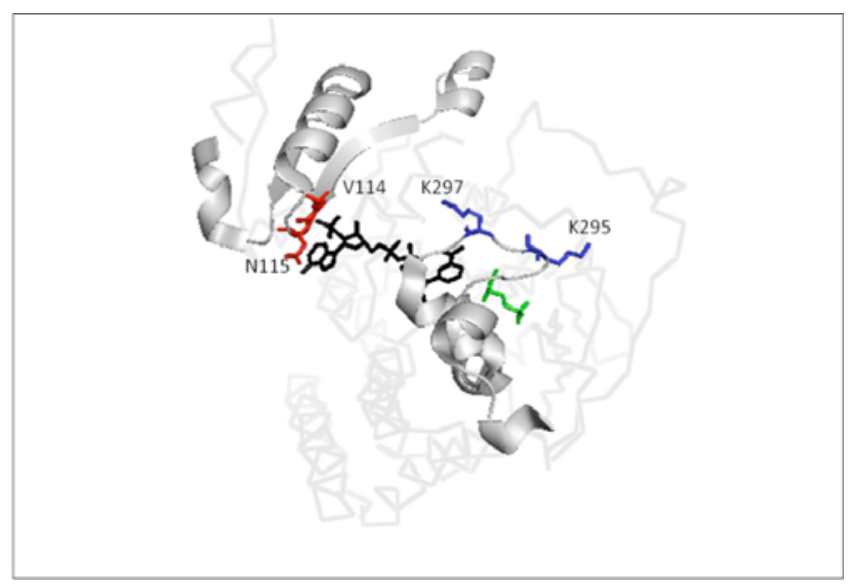

Figure 1. The three-dimensional structure of PfDXR in complex with an inhibitor and cofactor. Stick representation of a monomer of PfDXR with bound inhibitor FR900098 (an N-acetyl derivative of fosmidomycin) shown in green and NADPH in black, both denoted as stick models (pdb accession code: 3AUA [24]). Residues that are part of the catalytic hatch, K295 and K297 (coloured blue and represented as sticks), and residues involved in the binding of NADPH, V114 and N115 (coloured red and represented as sticks), were mutated to resemble EcDXR. The diagram was rendered using PyMOL [53].

\section{MATERIALS AND METHODS}

\section{Materials}

All reagents were obtained from Sigma Chemicals (USA), Roche Molecular Biochemicals (USA) or Merck Chemicals (Germany) unless otherwise stated. The plasmid encoding the codon optimized PfDXR was kindly donated by $\mathrm{Dr}$ H. Jomaa (Justus-Liebig-Universität Giessen, Germany). Table 1 gives an overview of the plasmids used in this study, including origins of replications, inducers and sources. For the purpose of this study, the protein produced from the codon harmonized PfDXR coding region will be named hPfDXR, and the protein produced from the codon optimized PfDXR coding region will be named oPfDXR.

\section{Codon Harmonisation of the Coding Region of PfDXR}

The codon harmonization algorithm used in this study was implemented as a PHP-script driven web interface and 
Table 1. Plasmids Encoding the Target Proteins of Interest, as well as the Molecular Chaperones and lac Repressor Investigated in this STUDY

\begin{tabular}{|c|c|c|c|}
\hline Plasmid & Description & Inducer & Source \\
\hline pQE31-oPfDXR & ColE1 ori, codon optimized coding region for PfDXR, Amp ${ }^{r}$ & $1 \mathrm{mM}$ IPTG & H Jomaa \\
\hline pQE30-hPfDXR & ColE1 ori, codon harmonised coding region for PfDXR, Amp ${ }^{\mathrm{r}}$ & $1 \mathrm{mM}$ IPTG & This study \\
\hline pQE30-hPfDXR- K295N, K297S & $\begin{array}{l}\text { ColE1 ori, codon harmonised coding region for PfDXR- } \\
\text { K295N, K297S, Amp }{ }^{r}\end{array}$ & $1 \mathrm{mM}$ IPTG & This study \\
\hline pQE30-hPfDXR- V114A, N115G & $\begin{array}{l}\text { ColE1 ori, codon harmonised coding region for PfDXR-V114A, } \\
\qquad \text { N115G, } \mathrm{Amp}^{\mathrm{r}}\end{array}$ & $1 \mathrm{mM}$ IPTG & This study \\
\hline pGro7 & p15A ori, GroEL-GroES, Cam ${ }^{\mathrm{r}}$ & $0.2 \%_{\mathrm{L}}-$ Arabinose & Takara Bio Inc. \\
\hline pKJE7 & p15A ori, DnaK-DnaJ-GrpE, Cam ${ }^{\mathrm{r}}$ & $0.2 \%_{\mathrm{L}}-$ Arabinose & Takara Bio Inc. \\
\hline pMRBAD-PfHsp70-1 & p15A ori, PfHsp70-1, Kan ${ }^{\mathrm{r}}$ & $0.2 \%_{\mathrm{L}}-$ Arabinose & A Shonhai \\
\hline pREP4 & p15A ori, lacI, $\operatorname{Kan}^{\mathrm{r}}$ & None & Qiagen \\
\hline
\end{tabular}

is available at (www.sami.org.za/equalize). This algorithm follows a codon frequency matching approach whereby codons are assigned on the basis of closest matching codon preference fraction when comparing the source and target codon preference tables. Each wild-type codon is substituted with a synonymous codon having the closest codon preference match between the source and target codon preference tables. This approach preserves the pause site profile of a wild-type gene in the synthetic gene designed for heterologous expression. Pause sites are predicted by calculating pausing propensity of a sliding window by applying a modified codon adaptation index (CAI) approach.

The coding region of PfDXR (AF111813) was harmonized using the highly expressed $E$. coli codon preference as target codon preference table. The Shine-Delgarno (SD) removal module of the website indicated no putative false start sequences in the harmonized version of the gene. The final step in the design pipeline was the removal of selected restriction sites required for downstream subcloning steps and subsequent addition of BamHI and SalI restriction sites to the $5^{\prime}$ and 3 ' ends to facilitate directional cloning. The harmonized version of the gene was synthesized by GENART (WWw.geneart.com).

\section{Construction of the Plasmid Containing the Codon Har- monized Coding Region of PfDXR}

The codon harmonised DXR coding region was assembled from synthetic oligonucleotides and/or PCR products by GENART. The fragment was inserted into a pGA15 vector $\left(\operatorname{Kan}^{\mathrm{r}}\right)$ using $K p n \mathrm{I}$ and $S a c \mathrm{I}$ restriction sites. The plasmid DNA was purified (Pure Yield ${ }^{\mathrm{TM}}$ Plasmid Midiprep, Promega) from transformed bacteria and the final 0804945DXR-pGA15 construct was verified by DNA sequencing. The harmonised coding region for PfDXR was amplified by polymerase chain reaction (PCR) from plasmid 0804945DXR-pGA15. The forward primer 5'-GGA TCC GGC GCT ATC AAG AAA C-3' and reverse primer 5'-GTC GAC TTA AGA GCT GTT GTG-3' were synthesised and purchased from IDT and incorporate BamHI and SalI restriction sites in the forward and reverse primers (underlined) respectively. The amplified product was then inserted into the pGEM $®-T$ (Promega) plasmid vector from which the PfDXR encoding segment was restricted using BamHI and SalI restriction enzymes and ligated into the pQE30 plasmid vector (Qiagen) $\left(\mathrm{Amp}^{\mathrm{r}}\right)$ via compatible overhangs. The integrity of the resultant pQE30-hPfDXR plasmid construct was confirmed by restriction analysis, as well as DNA sequencing.

\section{Site-directed Mutagenesis of the Coding Region for PfDXR}

Mutations were performed on codons encoding conserved residues identified in a multiple sequence alignment of full-length DXR homologues, including the N-terminal leader sequence of $P$. falciparum. The double amino acid substitutions generated in this study were K295N, K297S and V114A, N115G. The mutagenic primers, containing the relevant double amino acid substitutions, were used for PCRbased site-directed mutagenesis. The integrity of the resultant plasmid constructs (Table 1) was confirmed by DNA sequencing.

\section{Heterologous Production of PfDXR}

E. coli XL1 Blue cells were transformed with either pQE31-oPfDXR or pQE30-hPfDXR plasmid DNA. For coexpression studies, E. coli XL1 Blue cells previously transformed with the appropriate chaperone plasmid DNA (Table 1) were transformed with either $\mathrm{pQE} 31-\mathrm{oPfDXR}$ or $\mathrm{pQE} 30$ hPfDXR plasmid. Sterile LB broth $(25 \mathrm{ml})$ containing the appropriate antibiotic (Table 1) was inoculated from a single colony and incubated at $37^{\circ} \mathrm{C}$ with shaking for 12-16 hours. The culture was diluted into fresh broth containing the appropriate antibiotic to a final volume of $250 \mathrm{ml}$, and the culture was allowed to grow until mid-log phase $\left(\mathrm{OD}_{600} 0.3-\right.$ 0.4). Protein production was then monitored by the collection of hourly and overnight samples both in the absence and the presence of the protein inducers (Table 1). For coexpression studies, the chaperones were induced at mid-log 
phase $\left(\mathrm{OD}_{600} 0.3-0.4\right)$ and the PfDXR proteins were induced an hour later. The whole-cell extracts were normalized to $\mathrm{OD}_{600}$ and the appropriate aliquots were run on $12 \%$ SDSPAGE gels. The protein bands were visualized using Coomassie brilliant blue staining. Western analysis was performed to confirm production of the PfDXR, GroEL and PfHsp70 proteins using anti-His (Amersham Biosciences, USA), anti-GroEL (Sigma, USA) and anti-PfHsp70 antibodies respectively. Detection of these proteins was achieved using the ECL western-based chemiluminescence kit (Amersham Biosciences, USA) according to manufacturer's instructions.

\section{Purification of PfDXR}

Protein production was induced for 3 hours and overnight growth, following which cells were resuspended in $1 / 20^{\text {th }}$ culture volume lysis buffer (100 mM Tris, $\mathrm{pH} 8.0,300 \mathrm{mM}$ $\mathrm{NaCl}, 10 \mathrm{mM}$ imidazole, $1 \mathrm{mM}$ PMSF). Cells were frozen overnight, and thawed in the presence of $1 \mathrm{mg} / \mathrm{ml}$ lysozyme. Mild sonication was carried out and the cellular debris was removed by centrifugation at $16000 \mathrm{~g}$ for 20 minutes in a bench top centrifuge (Eppendorf, Germany). Aliquots of the supernatant and pellet were used to determine the solubility of the PfDXR proteins. For hPfDXR, hPfDXR-K295N, K297S and hPfDXR-V114A, N115G purifications, E. coli M15 cells containing pREP4 were transformed with each of the respective plasmids. For oPfDXR purification, E. coli XL1 Blue cells expressing pGro7 were transformed with pQE31-oPfDXR. Protein production was induced for 3 hours and soluble PfDXR was purified using a nickelchelating Sepharose fast flow matrix (Pharmacia Biotech, Sweden), using a competitive elution strategy (100 mM Tris, $300 \mathrm{mM} \mathrm{NaCl}, 500 \mathrm{mM}$ imidazole). The eluted protein was then buffer-exchanged into $100 \mathrm{mM}$ Tris- $\mathrm{HCl}(\mathrm{pH} 7.5)$ containing $300 \mathrm{mM} \mathrm{NaCl}$ via size exclusion chromatography at $4^{\circ} \mathrm{C}$ using a Sephadex G-25 Medium (Amersham Biosciences) column $(10 \mathrm{~cm} \times 1 \mathrm{~cm})$. For oPfDXR purification, additional steps were included to reduce the amount of copurified GroEL, whereby $25 \mathrm{mM}$ ATP and $0.5 \mathrm{ml}$ of prepared denatured protein were added to the soluble protein before affinity purification, as described by Rohman and Harrison-Lavoie [27]. The quantity of protein recovered was determined using the Bradford protein assay [28], with bovine serum albumin (BSA) as a standard, and the protein purity was qualitatively assessed using SDS-PAGE analysis.

\section{NADPH-dependent Enzyme Assay}

The assay was performed in a reaction mixture containing $100 \mathrm{mM}$ Tris- $\mathrm{HCl}(\mathrm{pH} 7.5), 1 \mathrm{mM} \mathrm{MnCl} 2,0.3 \mathrm{mM}$ deoxyxylulose phosphate (DXP), $0.3 \mathrm{mM} \mathrm{NADPH}$, in a final volume of $200 \mu 1$ [29]. The reaction was initiated by adding $20 \mu \mathrm{g} / \mathrm{ml}$ PfDXR or $2 \mu \mathrm{g} / \mathrm{ml}$ EcDXR enzyme and the oxidation of NADPH was monitored at $340 \mathrm{~nm}$ using a PowerWave $^{\mathrm{TM}}$ Microplate Spectrophotometer adjusted to $37^{\circ} \mathrm{C}$. Activity of DXR was expressed in units/mg of protein where 1 unit is defined as the amount of enzyme that causes oxidation of $1 \mu \mathrm{mol}$ of NADPH per minute. This experiment was done in triplicate for four separate batches of PfDXR protein. Lineweaver-Burk plots were used to calculate the kinetic constants for both EcDXR and PfDXR.

\section{RESULTS}

\section{The Levels of Soluble PfDXR Improved After Co- production of $\boldsymbol{E}$. coli and Malarial Chaperones}

SDS-PAGE and western analyses revealed that the oPfDXR protein (protein produced from the codon optimised coding region of PfDXR) was insoluble after overnight growth (Fig. 2A). In an attempt to enhance the solubility of PfDXR, the coding region was harmonized. However, hPfDXR (protein produced from the codon harmonised coding region of PfDXR) was not over-produced and resulted in decreased protein levels compared to oPfDXR (Fig. 2B). The codon harmonized coding region was less efficiently expressed than the codon optimized coding region, and PfDXR remained insoluble. The ability of heterologous molecular chaperones to enhance solubility was assessed and the coproduction of GroELS with oPfDXR resulted in the successful solubilization of $\sim 50 \%$ of the PfDXR protein (Fig. 3A), while co-production of DnaKJE did not enhance solubility (Fig. 3B). The solubility of hPfDXR was enhanced by the co-production of GroELS, and western analysis revealed that $\sim 80 \%$ of the protein was soluble (Fig. 4A). The amount of hPfDXR produced was considerably less than the amount of oPfDXR produced. In a similar manner to oPfDXR, coproduction of the DnaKJE family did not enhance the solubility of hPfDXR (Fig. 4B). Despite an enhancement in the levels of hPfDXR after co-production of the homologous molecular chaperone PfHsp70-1 (Fig. 5A), in comparison to GroEL, the protein was insoluble (Fig. 5B).

\section{Purification of PfDXR}

The largest yield of soluble oPfDXR was achieved during co-expression of GroELS, however, the purification was complicated by the co-elution of GroEL with PfDXR. Contaminating GroEL was effectively reduced by the addition of denatured protein to the soluble protein fraction during purification, but western analysis confirmed that oPfDXR was only partially purified as contaminating GroEL was still present (data not shown).

The tight control of gene expression was the final strategy employed to enhance expression and solubility of PfDXR. The transcription of the hPfdxr gene was halted by the presence of the lac repressor protein in the E. coli host expression system. Based on SDS-PAGE analysis, almost no target protein was observed before IPTG induction; whilst high levels of protein were evident after IPTG induction (Fig. 6A). The high levels of soluble protein (Fig. 6B, lane S) facilitated purification using nickel affinity chromatography. Most of the protein was present in fractions $10-12$ and the purity was assessed to be $\sim 90 \%$ using SDS-PAGE analysis; and western analysis confirmed the identity of Histagged PfDXR (Fig. 6B). In conclusion, between 2 and $4 \mathrm{mg}$ of functional hPfDXR target protein per litre of culture was purified using a lac repressor co-expression strategy that tightly controlled transcription.

\section{Kinetic analysis of PfDXR}

DXR catalyzes the second step of the MEP pathway whereby the substrate DOXP undergoes intramolecular rear- 

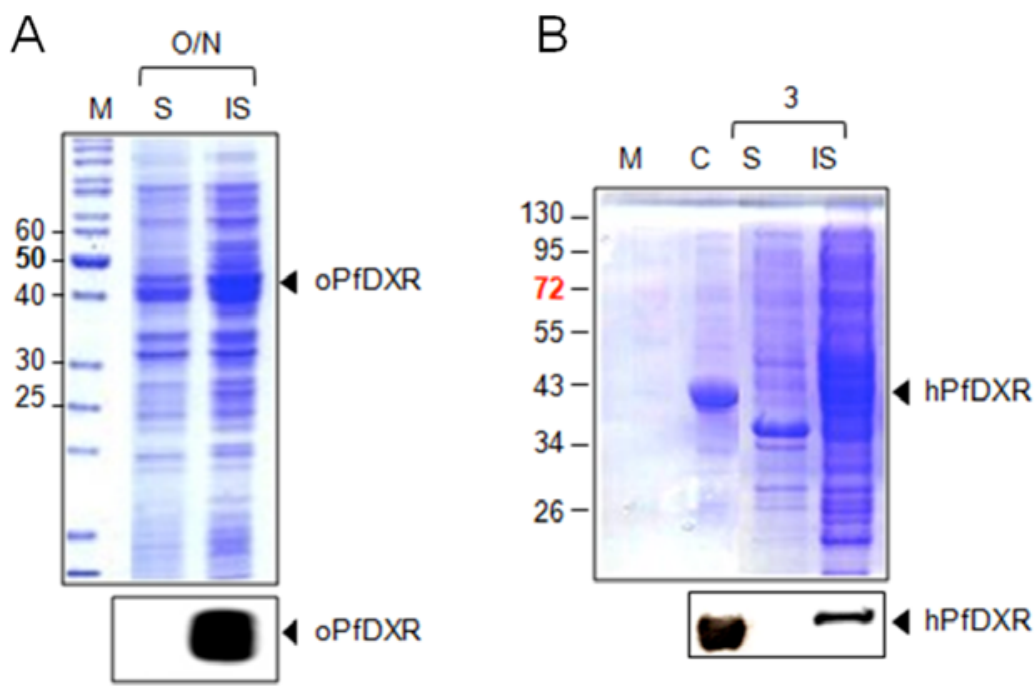

Figure 2. Analysis of PfDXR solubility in $\boldsymbol{E}$. coli cells. SDS-PAGE analysis of the solubility study of E. coli [pQE31-oPfDXR] after overnight induction $(\mathrm{O} / \mathrm{N})(\mathbf{A})$, and $E$. coli [pQE30-hPfDXR] after 3 hours of induction $(\mathbf{B})$, where protein samples were separated into the soluble (S) and insoluble (IS) fractions after cell lysis. Western analysis was performed using anti-His antibodies for PfDXR. M = marker (kDa), $\mathrm{C}=$ purified EcDXR of $44.6 \mathrm{kDa}$, oPfDXR $=48.4 \mathrm{kDa}$, and $\mathrm{hPfDXR}=46.7 \mathrm{kDa}$.
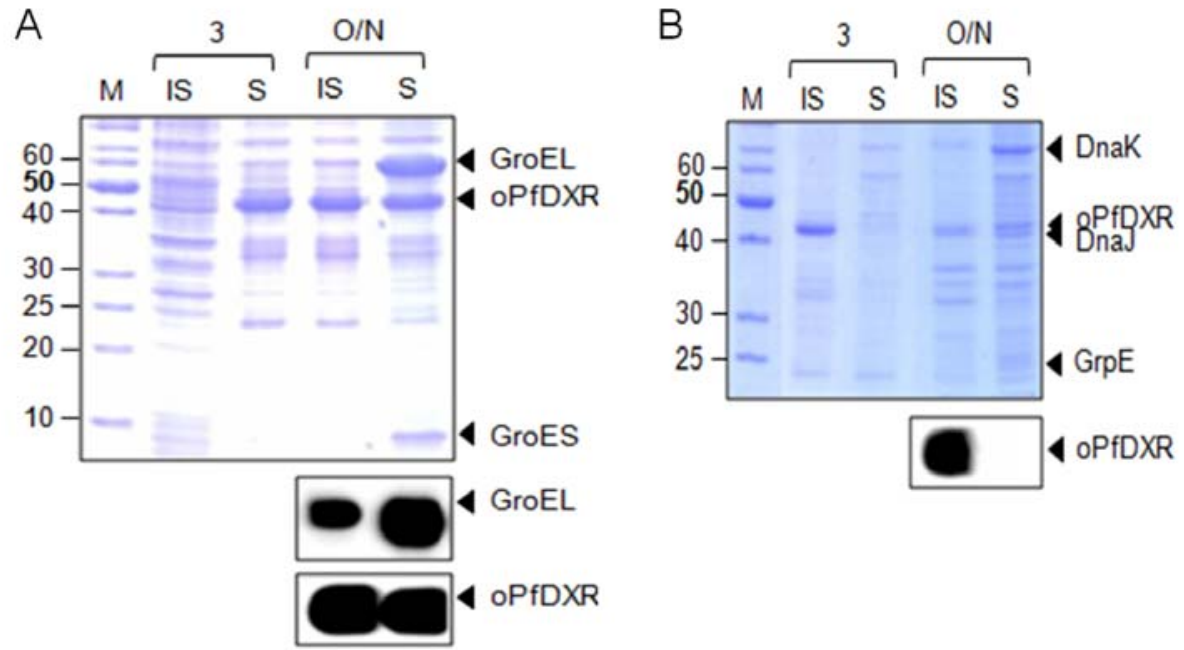

Figure 3. Analysis of oPfDXR solubility after co-expression with the $E$. coli molecular chaperones DnaKJE and GroEL-ES. SDSPAGE analysis of the solubility study of E. coli [pQE31-oPfDXR][pGro7] (A), and E. coli [pQE31-oPfDXR][pKJE7] (B), where oPfDXR was co-expressed with GroEL-ES and DnaKJE chaperones respectively. Protein samples were separated into the soluble (S) and insoluble (IS) fractions after cell lysis. Western analysis was performed using anti-His antibodies for PfDXR and anti-GroEL antibodies for the detection of GroEL. $\mathrm{M}=$ marker $(\mathrm{kDa}), \mathrm{DnaK}=70 \mathrm{kDa}, \mathrm{GroEL}=60 \mathrm{kDa}$, DnaJ $=40 \mathrm{kDa}, \mathrm{GrpE}=25 \mathrm{kDa}, \mathrm{GroES}=10 \mathrm{kDa}$.

rangement and reduction to result in MEP; this reaction step utilizes NADPH, as well as a divalent cation [30]. DOXP is not only an intermediate in the biosynthesis of isopentenyl pyrophosphate and dimethylallyl diphosphate; it is also involved in the biosynthesis of thiamin in vitamin B1 synthesis and pyridoxine in vitamin B6 synthesis [31, 32].

Enzymatic activity was assessed in the presence of various divalent cations. Literature demonstrates that numerous cofactor studies have been performed for other DXR enzymes [33, 34, 35], however to date, cofactor studies using PfDXR have only been examined in silico [25]. Enzyme activity was quantified in the presence of three cofactors; PfDXR was only able to utilize $\mathrm{Mn}^{2+}$ and $\mathrm{Mg}^{2+}$ as a divalent cation but not $\mathrm{Co}^{2+}$, which resulted in only trace amounts of enzyme activity (Table 2). EcDXR was able to utilize all three cofactors, with $\mathrm{Mn}^{2+}$ resulting in the greatest activity (Table 2).

After the purification of PfDXR, an enzymatic activity of $1.04 \pm 0.04 \mu \mathrm{mol} / \mathrm{min} / \mathrm{mg}$ was reported for PfDXR, in the presence of $\mathrm{Mn}^{2+}$, while EcDXR demonstrated a 10-fold greater activity of $9.15 \pm 0.05 \mu \mathrm{mol} / \mathrm{min} / \mathrm{mg}$, which is similar to that reported in the literature [29]. In order to assess the 
Table 2. Summary of PfDXR-K295N, K297S and PfDXR-V114A, N115G Kinetic Parameters, $V_{\max }(\mu \mathrm{mol} / \mathrm{min} / \mathrm{mg}), \mathrm{K}_{\mathrm{m}}(\mathrm{mM})$ and $k_{\text {cat }}\left(\min ^{-1}\right)$, as well as the Divalent Cation Preferences for PfDXR and EcDXR

\begin{tabular}{|c|c|c|c|c|}
\hline & EcDXR & PfDXR & PfDXR-K295N, K297S & PfDXR-V114A, N115G \\
\hline \hline $\mathrm{Mg}^{2+}(\mu \mathrm{mol} / \mathrm{min} / \mathrm{mg})$ & $5.94 \pm 0.19$ & $0.65 \pm 0.02$ & & \\
\hline $\mathrm{Co}^{2+}(\mu \mathrm{mol} / \mathrm{min} / \mathrm{mg})$ & $3.98 \pm 0.13$ & $0.30 \pm 0.03$ & & \\
\hline $\mathrm{Mn}^{2+}(\mu \mathrm{mol} / \mathrm{min} / \mathrm{mg})$ & $9.15 \pm 0.05$ & $1.04 \pm 0.04$ & & $0.369 \mu \mathrm{mol} / \mathrm{min} / \mathrm{mg}$ \\
\hline $\mathrm{V}_{\max }$ & $11.04 \mu \mathrm{mol} / \mathrm{min} / \mathrm{mg}$ & $1.33 \mu \mathrm{mol} / \mathrm{min} / \mathrm{mg}$ & $0.59 \mu \mathrm{mol} / \mathrm{min} / \mathrm{mg}$ & $0.461 \mathrm{mM}$ \\
\hline $\mathrm{K}_{\mathrm{m}}$ & $0.294 \mathrm{mM}$ & $0.067 \mathrm{mM}$ & $0.245 \mathrm{mM}$ & $0.572^{-\mathrm{s}}$ \\
\hline $\mathrm{k}_{\mathrm{cat}}$ & $17.12^{-\mathrm{s}}$ & $2.063^{-\mathrm{s}}$ & $0.915^{-\mathrm{s}}$ & 0.0022 \\
\hline $\mathrm{k}_{\mathrm{cat}} / \mathrm{K}_{\mathrm{m}}$ & 0.058 & 0.031 & 0.0037 & \\
\hline
\end{tabular}

A

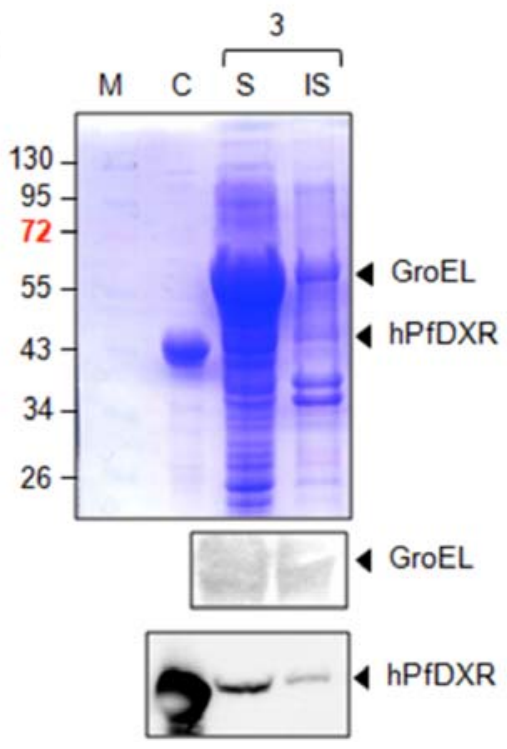

B

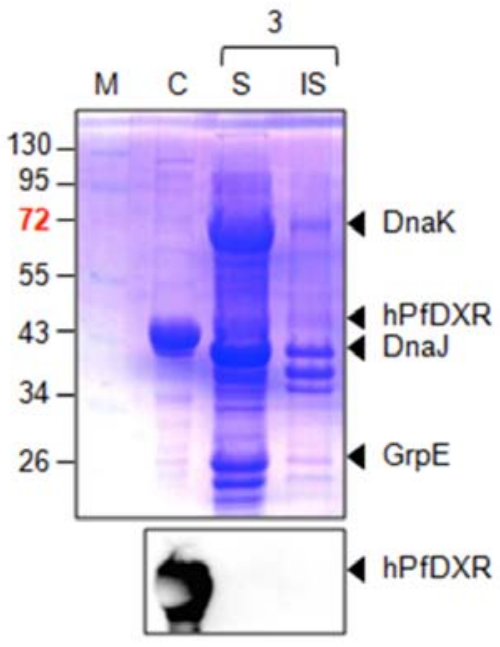

Figure 4. Analysis of hPfDXR solubility after co-expression with the $E$. coli molecular chaperones DnaKJE and GroEL-ES. SDSPAGE analysis of the solubility study of E. coli [pQE30-hPfDXR][pGro7] (A), and E. coli[pQE30-hPfDXR][pKJE7] (B), where hPfDXR was co-expressed with GroEL-ES and DnaKJ-GrpE chaperones respectively. Protein samples were separated into the soluble (S) and insoluble (IS) fractions after cell lysis. Western analysis was performed using anti-His antibodies for PfDXR and anti-GroEL antibodies for the detection of GroEL. $\mathrm{M}=$ marker $(\mathrm{kDa}), \mathrm{C}=$ control (purified EcDXR of $44.6 \mathrm{kDa}$ ), DnaK $=70 \mathrm{kDa}$, GroEL $=60 \mathrm{kDa}, \mathrm{DnaJ}=40 \mathrm{kDa}$, $\mathrm{GrpE}=25 \mathrm{kDa}, \mathrm{GroES}=10 \mathrm{kDa}$.

kinetics of PfDXR, $\mathrm{V}_{\max }, \mathrm{K}_{\mathrm{m}}$ and $\mathrm{k}_{\mathrm{cat}}$ values were calculated. The kinetics were determined by varying the substrate (DXP) concentrations and not the NADPH concentrations. $\mathrm{K}_{\mathrm{m}}$ values that have been reported in the literature for EcDXR range from $0.0031 \mathrm{mM}$ to $0.25 \mathrm{mM}$ [36]. In this work, we report a $\mathrm{K}_{\mathrm{m}}$ value of $0.294 \mathrm{mM}$ for EcDXR and $0.067 \mathrm{mM}$ for PfDXR; these values being consistent with the literature for EcDXR, while there appears to be no kinetic parameters reported for PfDXR [36]. The maximum velocity of the reaction whereby DXR converts DXP to MEP was determined to be $1.33 \mu \mathrm{mol} / \mathrm{min} / \mathrm{mg}$ and $11.04 \mu \mathrm{mol} / \mathrm{min} / \mathrm{mg}$ for PfDXR and EcDXR respectively (Table 2). In the case of both enzymes, the average specific activity is approximately equal to the maximum velocity of the reaction. This is due to sub-saturating levels of substrate in the assay, meaning that the maximal velocity of the reaction is not reached.
The overall turnover rate $\left(\mathrm{k}_{\mathrm{cat}}\right)$ was quantified for both enzymes. The literature reports values for other DXR enzymes in the range of 0.04 to $38 \mathrm{~s}^{-1}$ for organisms including Mycobacterium tuberculosis, Synechocystis sp., Arabidopsis thaliana and E. coli [37-40]. In this work, values of 17.12 and 2.063 units per second $\left(\mathrm{s}^{-1}\right)$ were reported for EcDXR and PfDXR respectively. The catalytic efficiency of EcDXR is only 2-fold greater than that of PfDXR, due to the significantly greater substrate affinity of PfDXR.

\section{Rational Protein Engineering of PfDXR}

Numerous site-directed mutagenesis studies have been undertaken on DXR (almost exclusively on E. coli) including residues responsible for cofactor binding, structural residues that support the catalytic residues, residues that interact with 
A
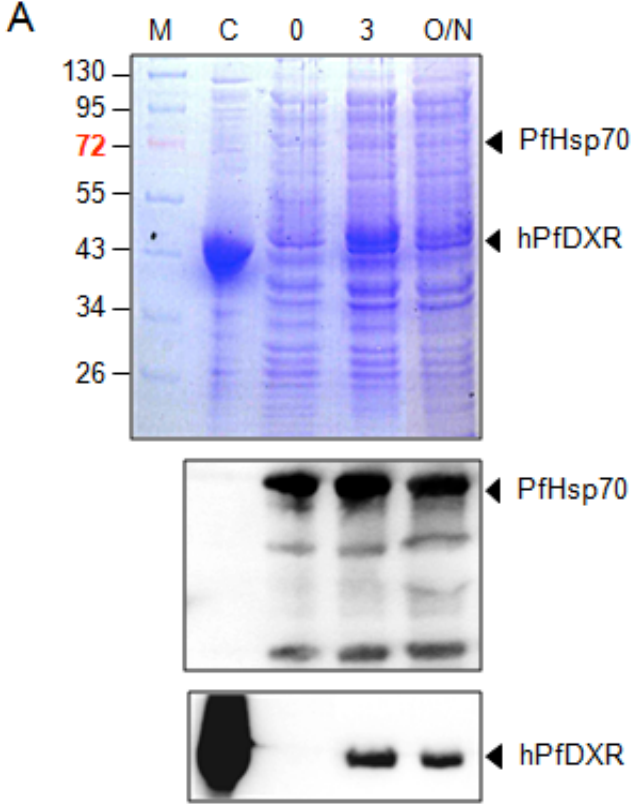

B

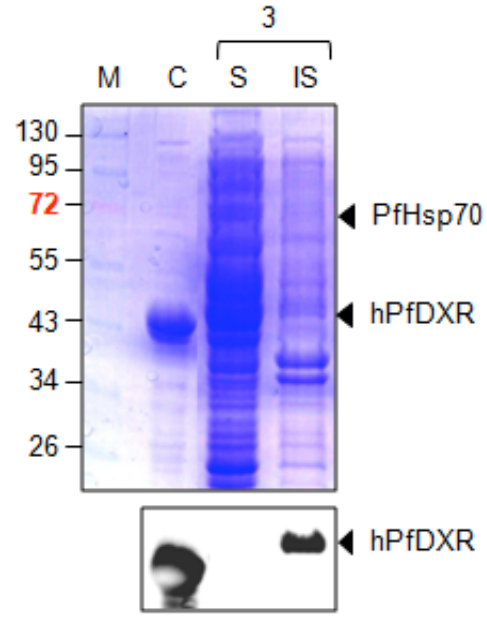

Figure 5. Analysis of hPfDXR induction and solubility after coexpression with the malarial chaperone PfHsp70-1. SDS-PAGE analysis of the induction study of $E$. coli [pQE30-hPfDXR] [pMRBAD-PfHsp70-1] (A), where hPfDXR was co-expressed with PfHsp70-1. Whole cells were monitored before ${ }_{L}$-arabinose and IPTG induction (0), after 3 hours of induction (3) and after overnight induction $(\mathrm{O} / \mathrm{N})$. SDS-PAGE analysis of the solubility study of E. coli [pQE30-hPfDXR][pMRBAD-PfHsp70-1] (B), where protein samples were separated into the soluble $(\mathrm{S})$ and insoluble (IS) fractions after cell lysis. Western analysis was performed using anti-His antibodies for PfDXR. $\mathrm{M}=$ marker $(\mathrm{kDa}), \mathrm{C}$ $=$ control (purified EcDXR = 44.6 kDa), PfHsp70-1 = $70 \mathrm{kDa}$.

the substrate and residues of the catalytic hatch $[22,23,25$, 41- 43], however no literature is available for PfDXR. In this study, two double amino acid substitutions were carried out on PfDXR to generate a modified PfDXR in which the active site more closely resembled that of EcDXR. One of the double amino acid substitutions targeted the NADPH binding residues of PfDXR, V114 and N115, to resemble the EcDXR equivalents of A35 and G36. This mutation resulted in a 2fold decrease in maximum velocity of the reaction $\left(\mathrm{V}_{\max }\right)$,
A

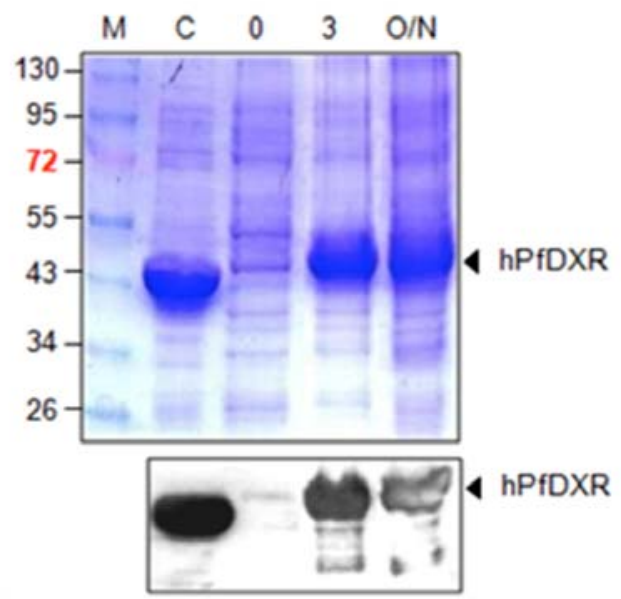

B

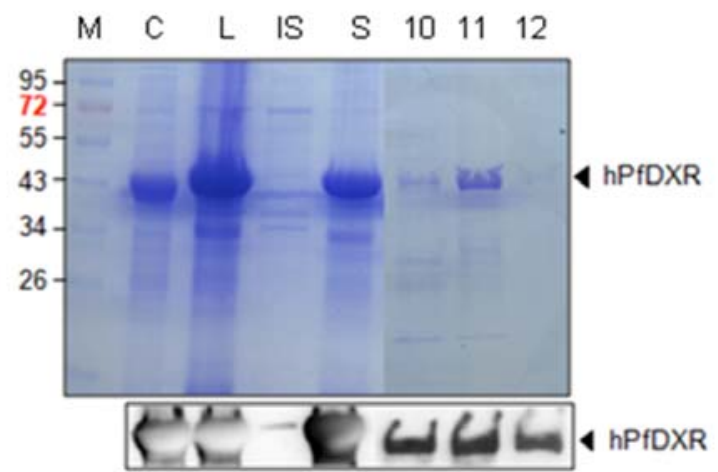

Figure 6. Purification by nickel affinity chromatography of hPfDXR produced during co-expression with the lac repressor protein. SDS-PAGE analysis of the induction study of $E$. coli [pQE30-hPfDXR][pREP4] (A), where hPfDXR was co-expressed with lac repressor protein. Whole cells were monitored before IPTG induction (0), after 3 hours of induction (3) and after overnight induction $(\mathrm{O} / \mathrm{N})$. Analysis of protein before and after optimized purification of hPfDXR $(\mathbf{B})$, protein samples were separated into total lysate after sonication (L), the soluble (S) and insoluble (IS) fractions after cell lysis to determine solubility. Fractions $10-$ 12 contained the highest concentration of PfDXR obtained after competitive gradient elution with $500 \mathrm{mM}$ imidazole. The concentration of protein in fraction $11=0.8 \mathrm{mg} / \mathrm{ml}$. Western analysis was performed using anti-His antibodies for PfDXR. $\mathrm{M}=$ marker $(\mathrm{kDa}), \mathrm{C}=$ control (purified EcDXR of $44.6 \mathrm{kDa}$ ).

and a 4-fold increase in $K_{m}$ (Table 2). Interestingly, these mutations increased the $\mathrm{K}_{\mathrm{m}}$ to values resembling that of EcDXR. Substituting the large basic residues K295 and K297, unique to species of Plasmodium and located in the catalytic hatch region, to small polar asparagine and serine equivalents found in EcDXR, resulted in a 4-fold decrease in maximum reaction velocity and 7-fold increase in the $K_{m}$ (Table 2). A decreased ability to turnover substrate by both PfDXR-K295N, K297S and PfDXR-V114A, N115G stresses the importance of these residues in securing the substrate and cofactor. 


\section{DISCUSSION}

Codon bias can be problematic during the production of heterologous proteins in E. coli, and codon harmonization was one of the approaches taken in this study to produce soluble PfDXR. The method used for harmonization was such that there was preservation of the pause site profile of the native sequence; with the intention that the correct arrangements of the protein secondary structures would be maintained. Analysis of the sequence alignment of the codon optimized versus the codon harmonised coding regions for PfDXR revealed that they share $79 \%$ sequence identity, while the protein sequence was preserved. Codon harmonization resulted in a decrease in the amount of PfDXR produced; however neither codon optimization nor harmonization resulted in the production of soluble PfDXR, confirming the recalcitrant nature of the PfDXR protein.

Often the host cell's chaperone system becomes overloaded during heterologous protein production, limiting the amount of soluble protein that can be produced [5]. The over-production of molecular chaperones has been used successfully for a wide range of proteins; however it is difficult to predict which chaperone family will recognise a particular protein. E. coli GroELS recognised PfDXR as a substrate and significantly enhanced its solubility, while DnaKJE failed to enhance the solubility of the protein. Due to the size of the cavity formed by GroEL, this chaperone family has an upper size limit for substrates of $\sim 60 \mathrm{kDa}$ [44], therefore allowing PfDXR (47 kDa) to act as a substrate.

The E. coli chaperones did not completely solubilise the PfDXR proteins and thus homologous molecular chaperones were used. The production of hPfDXR was enhanced by the co-expression of the $P$. falciparum homologue of DnaK (PfHsp70-1), however western analysis confirmed that all of the target protein was essentially insoluble. PfHsp70-3 (NP 701211) and PfHsp70-y (MAL13P1.540) are potentially localized to the apicoplast [45], but PhHsp70-y is unlikely to be an apicoplast protein due to the presence of a KDEL ER retrieval sequence [46], and the PfHsp70 used in this study was cytosolic PfHsp70-1 [45] and therefore may not be suitable when trying to express the apicoplast target protein PfDXR. There is evidence to suggest that PfHsp70-1 interacts with numerous apicoplast proteins [47, 48]. Based on the results in this study, however, the most probable molecular chaperone folding partner may be the $P$. falciparum homologue of GroEL (PfHsp60) localised in the apicoplast [49].

The most successful strategy to improve expression and solubility was the use of the lac repressor protein, which halts basal transcription in the E. coli host expression system, and this system was used for the purification of hPfDXR. It is possible that this system led to the greatest control of the levels of PfDXR, and the enzyme was induced at the correct time during growth of the cells to enable the production of soluble protein. The purity of hPfDXR was assessed to be $\sim 90 \%$ using SDS-PAGE analysis and the yield of $2-4 \mathrm{mg}$ of hPfDXR per litre of culture was 8 to 10 -fold higher than previously reported yields [50].

After the purification of PfDXR, the enzymatic activity was assessed in order to determine whether folded, func- tional protein was produced. In this study, PfDXR demonstrated a specific activity 10 -fold less than that of EcDXR, which is similar to literature [29]. According to an online enzyme database [36], specific activities of 19.5 and 5.6 $\mu \mathrm{mol} / \mathrm{min} / \mathrm{mg}$ for Zymomonas mobilis and Arabidopsis thaliana DXR (ZmDXR and AtDXR) respectively have been reported [51, 52]. While one group has reported a specific activity for $P$. falciparum DXR, the units were not specified and protein purity was only $80 \%$ [29].

Cofactor studies of PfDXR have been examined in silico [25]. Takenoya [35] examined the effect of cofactors on the activity of the hyperthermophile Thermotoga maritima DXR (tDXR) and the enzyme could utilize either $\mathrm{Mn}^{2+}$ or $\mathrm{Mg}^{2+}$ as a divalent cation, but not $\mathrm{Co}^{2+}$. Previous studies concluded that the EcDXR enzyme could utilize $\mathrm{Mn}^{2+}, \mathrm{Co}^{2+}$ and $\mathrm{Mg}^{2+}$, with $\mathrm{Mn}^{2+}$ generating the highest activity [34], and the results were confirmed in this study. PfDXR was only able to utilize $\mathrm{Mn}^{2+}$ or $\mathrm{Mg}^{2+}$ as a divalent cation, but not $\mathrm{Co}^{2+}$, which is similar to tDXR.

The results of this study emphasize the significance of the basic, large K295 and K297 residues present in the catalytic hatch of PfDXR ; the substitution of these residues resulted in a considerable increase in the $\mathrm{K}_{\mathrm{m}}$, suggesting a decrease in affinity for the substrate. Substitution of these residues on EcDXR may enhance the substrate affinity of this enzyme. PfDXR-V114A,N115G also displayed an increase in the $\mathrm{K}_{\mathrm{m}}$ relative to the unmodified PfDXR, which cannot be accounted for in terms of a decrease in affinity for the substrate, since these residues are involved in cofactor binding and not directly involved in substrate recognition and catalysis. Mutation of these residues may have had an effect on the overall structure of the enzyme. However, the kinetic parameters should have been measured in the presence of varying NADPH concentrations in order to determine the effect of these mutations on cofactor binding. PfDXRK295N,K297S and PfDXR-V114A,N115G demonstrated a decreased ability to turnover substrate by 4 -fold and 2-fold, respectively, compared to PfDXR. The difference in turnover number between these proteins could be due to the maintenance of the catalytic hatch in PfDXR-V114A, N115G and a reduction in the efficiency of binding NADPH while maintaining substrate affinity.

PfDXR-K295N, K297S demonstrated a 24-fold decrease in catalytic efficiency due to a decrease in substrate affinity coupled to a decreased turnover rate. This reduction may be due to substrate being unable to enter the active site as easily or due to the lack of the original lysine residues which may form additional contacts with the substrate, enhancing affinity. PfDXR-V114A, N115G shares a similar $\mathrm{K}_{\mathrm{m}}$ to EcDXR, and the reduced catalytic efficiency is a result of the reduced turnover number.

\section{CONCLUSION}

In conclusion, the results of this study show that the influence of various strategies to improve solubility cannot be predicted, but must be optimized for each particular heterologous protein. Once a successful purification strategy had been developed, the importance of residues in the catalytic hatch and involved in cofactor binding could be studied using site-directed mutagenesis. The recent successful crys- 
tallization of PfDXR in the presence and absence of an inhibitor [24], coupled to the structural information that comes from rational protein engineering, will aid in the development of novel inhibitors. The PfDXR protein produced in this study will be used for ongoing research investigating novel inhibitors of this enzyme.

\section{CONFLICT OF INTEREST}

The authors confirm that this article content has no conflicts of interest.

\section{ACKNOWLEDGEMENTS}

The authors gratefully acknowledge $\mathrm{Dr} H$. Jomaa (Justus-Liebig-Universität Giessen, Germany) for the plasmid encoding the codon optimized PfDXR. We also thank Dr A. Shonhai for providing the plasmid encoding PfHsp70. This research was funded by National Research Foundation Grant awarded to AB (Thuthuka Programme) and a SAMI Grant awarded to GLB. LSS was awarded a post-doctoral bursary by the South African Malaria Initiative programme; JG was awarded a PhD bursary by SAMI and National Research Foundation and HJ was awarded an Honours bursary by Rhodes University.

\section{ABBREVIATIONS}

PfDXR $=\begin{aligned} & \text { Plasmodium falciparum 1-deoxy-D- } \\ & \text { xylulose-5-phosphate reductoisomerase }\end{aligned}$
EcDXR $=\begin{aligned} & \text { Escherichia coli } 1 \text {-deoxy-D-xylulose-5- } \\ & \text { phosphate reductoisomerase }\end{aligned}$

$\mathrm{Hsp} 70=$ heat shock protein 70.

\section{REFERENCES} Pharm. Des., 2007, 13, 1161-1177. Odom, A.R., Van Voorhis, W.C. Functional genetic analysis of the Plasmodium falciparum deoxyxylulose 5-phosphate reductoisomerase gene. Mol. Biochem. Parasitol., 2010, 170, 108-111. World
Health
Organization
(2010)
Malaria.

http://www.who.int/topics/malaria/en/

ity on recombinant expression of Plasm, M. Prediction of solubilcyte membrane protein 1 domains in Escherichia coli. Malaria J., 2006, 5, 52 .

Carrió, M.M., Villaverde, A. Construction and deconstruction of bacterial inclusion bodies. J. Biotechnol., 2002, 96, 3-12.

Georgiou, G., Valax, P. Expression of correctly folded proteins in Escherichia coli. Curr. Opin. Biotechnol., 1996, 7, 190-197.

Kohda, J., Endo, Y., Okumura, N., Kurokawa, Y., Nishihara, K., Yanagi, H., Yura, T., Fukuda, H., Kondo, A. Improvement of productivity of active form of glutamate racemase in Escherichia coli by coexpression of folding accessory proteins. Biochem. Eng., 2002, 10, 39-45.

8] Mehlin, C., Boni, E., Buckner, F.S., Engel, L., Feist, T., Gelb, M.H., Haji, L., Kim, D., Liu, C., Mueller, N., Myler, P.J., Reddy, J.T., Sampson, J.N., Subramanian, E., Van Voorhis, W.C., Worthey, E., Zucker, F., Hol, W.J.G. (2006) Heterologous expression of proteins from Plasmodium falciparum: Results from 1000 genes. Mol. Biochem. Parasitol., 2006, 148, 144-160.

Flick, K., Ahuja, S., Chene, A., Bejarano, M., Chen, Q. Optimized expression of Plasmodium falciparum erythrocyte membrane protein 1 domains in Escherichia coli. Malaria J., 2004, 3, 50.
Narum, D.L., Kumar, S., Rogers, W.O., Fuhrmann, S.R., Liang, H., Oakley, M., Taye, A., Sim, B.K.L., Hoffman, S.L. Codon optimization of gene fragments encoding Plasmodium falciparum merzoite proteins enhances DNA vaccine protein expression and immunogenicity in Mice. Infect. Immun., 2001, 69, 7250-7253.

Baca, A.M., Hol, W.G.J. Overcoming codon bias: A method for high-level overexpression of Plasmodium and other AT-rich parasite genes in Escherichia coli. Int. J. Parasitol., 2000, 30, 113-118. Nicoll, W.S., Botha, M., McNamara, C., Schlange, M., Pesce, E.R., Boshoff, A., Ludewig, M.H., Zimmermann, R., Cheetham, M.E., Chapple, J.P., Blatch, G.L. Cytosolic and ER J-domains of mammalian and parasitic origin can functionally interact with DnaK. Int. J. Biochem. Cell Biol., 2007, 39, 736-751.

Angov, E., Hillier, C.J., Kincaid, R.L., Lyon, J.A. Heterologous protein expression is enhanced by harmonizing the codon usage frequencies of the target gene with those of the expression host. PLOS ONE., 2008, 3, 2189.

Matambo, T.S., Odunuga, O.O., Boshoff, A., Blatch, G.L. Overproduction, purification, and characterization of the Plasmodium falciparum heat shock protein 70. Protein Expr. Purif., 2004, 33, 214-222.

Birkholtz, L., Blatch, G.L., Coetzer, T., Hoppe, H., Human, E., Morris, E., Ngcete, Z., Oldfield, L., Roth, R., Shonhai, A., Stephens, L.L., Louw, A.I. Heterologous expression of plasmodial proteins for structural studies and functional annotation. Malaria J., 2008, 7, 197.

Ahn, T., Yang, S., Yun, C. Enhanced expression of human cytochrome P450 1A2 by co-expression with human molecular chaperone Hsp70. Toxicol. Lett., 2004, 153, 267-272.

Schrodel, A., Volz, J., de Marco, A. Fusion tags and chaperone coexpression modulate both the solubility and the inclusion body features of the recombinant CLIPB14 serine protease. J. Biotechnol. 2005, 120, 2-10.

Szabo, A., Langer, T., Schröder, H., Flanagan, J., Bukau, B., Hartl, F.-U. The ATP hydrolysis-dependent reaction cycle of the Escherichia coli Hsp70 system- DnaK, DnaJ and GrpE. Proc. Natl. Acad. Sci. U. S. A., 1994, 91, 10345-10349.

Rüdiger, S., Germoth, L., Schnieider-Mergener, J., Bakau, B. Substrate specificity of the DnaK chaperones determined by screening cellulose-bound peptide libraries. EMBO J., 1997, 16, 1501-1507.

Weissman, J.S., Hohl, C.M., Kovalenko, O., Kashi, Y., Chen, S., Braig, K., Saibil, H.R., Fenton, W.A., Horwich, A.L. Mechanism of GroEL action: productive release of polypeptide from a sequestered position under GroES. Cell., 1995, 83,577-587.

Burston, S.G., Clarke, A.R. Molecular chaperones: physical and mechanistic properties. Essays Biochem., 1995, 29, 125-136.

Reuter, K., Sanderbrand, S., Jomaa, H., Wiesner, J., Steinbrecher, I., Beck, E., Hintz, M., Klebe, G., Stubbs, M.T. Crystal structure of 1-deoxy-D-xyulose-5-phosphate reductoisomerase, a crucial enzyme in the non-mevalonate pathway of isoprenoid biosynthesis $J$. Biol. Chem., 2002, 277, 5378-5384.

[23] Yajima, S., Nonaka, T., Kuzuyama, T., Seto, H., Ohsawa, K. Crystal structure of 1-deoxy-D-xylulose 5-phosphate reductoisomerase complexed with cofactors: implications of a flexible loop movement upon substrate binding. J. Biochem., 2002, 131, 313317.

[24] Umeda, T., Tanaka, N., Kusakabe, Y., Nakanishi, M., Kitade, Y., Nakamura, K.T. Molecular basis of fosmidomycin's action on the human malaria parasite Plasmodium falciparum. Sci. Reports., 2011, 1, 9: DOI:10.1038/srep00009.

[25] Singh, N., Chevé, G., Avery, A., McCurdy, C.R. Comparative protein modeling of 1-deoxy-d-xylulose-5-phosphate reductoisomerase enzyme from Plasmodium falciparum: A potential target for antimalarial drug discovery. J. Chem. Inf. Model., 2006, 46, 1360-1370.

[26] Goble, J.L., Adendorff, M.R., de Beer, T.A.P., Stephens, L.L., Blatch, G.L. The malarial drug target Plasmodium falciparum 1deoxy-D-xylulose-5-phosphate reductoisomerase (PfDXR): Development of a 3-D Model for identification of novel, structural and functional features and for inhibitor screening. Prot. Pept. Lett., 2010,17, 109-120.

[27] Rohman, M., Harrison-Lavoie, K.J. Separation of co purifying GroEL from glutathione-S-transferase fusion proteins. Protein Expr. Purif., 2000, 20, 45-47. 

of microgram quantities of protein utilizing the principle of protein-dye binding. Anal. Biochem., 1976, 72, 248-254.

[29] Jomaa, H., Wiesner, J., Sanderbrand, S., Altincicek, B., Weidemeyer, C., Hintz, M., Türbachova, I., Eberl, M., Zeidler, J., Lichtenthaler, H.K., Soldati, D., Beck, E. Inhibitors of the nonmevalonate pathway of isoprenoid biosynthesis as antimalarial drugs. Science, 1999, 285, 1573-1576

[30] Kuzuyama, T., Shimizu, T., Takahashi, S., Seto, H. Fosmidomycin, a specific inhibitor of 1-deoxy-D-xylulose 5-phosphate reductoisomerase in the nonmevalonate pathway for terpenoid biosynthesis. Tetrahedron Lett., 1998, 39, 7913-7916.

[31] Hill, R.E., Himmeldirk, K., Kennedy, I.A., Pauloski, R.M., Sayer, B.G., Wolf, E., Spenser, I.D. A 13C NMR investigation of the biosynthesis of pyridoxol in Escherichia coli J. Biol. Chem., 1996, 271, 30426-30435.

[32] Cane, D.E., Du, S., Spenser, I.D. Biosynthesis of vitamin B B $_{6}$ Origin of the oxygen atoms of pyridoxol phosphate. J. Am. Chem. Soc., 2000, 122, 4213-4214.

[33] Argyrou, A., Blanchard, J.S. Kinetic and chemical mechanism of Mycobacterium tuberculosis 1-deoxy-D-xylulose-5-phosphate isomeroreductase. Biochemistry., 2004, 43, 4375-4384.

[34] Takahashi, S., Kuzuyama, T., Watanabe, H., Seto, H. A 1-deoxyD-xyulose 5-phosphate reductoisomerase catalyzing the formation of 2-C-methyl-D-erythritol 4-phosphate in an alternative nonmevalonate pathway for terpenoid biosynthesis. Proc. Natl. Acad. Sci. U. S. A., 1998, 95, 9879-9884.

[35] Takenoya, M., Ohtaki, A., Noguch,i K., Endo, K., Sasaki, Y., Ohsawa, K., Yajima, S., Yohda, M. Crystal structure of 1-deoxy-Dxylulose 5-phosphate reductoisomerase from the hyperthermophile Thermotoga maritima for insights into the coordination of conformational changes and an inhibitor binding. J. Struct. Biol., 2010, 170, 532-539.

[36] Chang, A., Scheer, M., Grote, A., Schomburg, I., Schomburg, D. BRENDA, AMENDA and FRENDA the enzyme information system: new content and tools in 2009. Nucl. Acids Res., 2009, 37, 588-592.

[37] Fernandes, R.P.M., Phaosiri, C., Proteau, P.J. Mutation in the flexible loop of 1-deoxy-D-xylulose 5-phosphate reductoisomerase broadens substrate utilization. Arch. Biochem. Biophys., 2006, 444,159-64.

[38] Henriksson, L.M., Björkelid, C., Mowbray, S.L., Unge, T. The 1.9 A resolution structure of Mycobacterium tuberculosis 1-deoxy-Dxylulose 5-phosphate reductoisomerase, a potential drug target. Acta Crystallogr. D Biol. Crystallogr., 2006, 62, 807-813.

[39] Fox, D.T., Poulter, C.D. Mechanistic studies with 2-C-methyl-derythritol 4-phosphate synthase from Escherichia coli. Biochemis$\operatorname{try}(\mathrm{N}$. Y.), 2005, 44, 8360-8368.

[40] Walker, J.R., Poulter, D.C. Synthesis and evaluation of 1-deoxyd-xylulose 5-phosphate analogues as chelation-based inhibitors of methylerythritol phosphate synthase. J. Org. Chem., 2005, 70, 9955-9959.

Fernandes, R.P.M.., Phaosiri, C., Proteau, P.J. Mutation in the flexible loop of 1-deoxy-D-xylulose 5-phosphate reductoisomerase broadens substrate utilization. Archiv. Biochem. Biophys. 2005, 444, 159-164.

Steinbacher, S., Kaiser, J., Eisenreich, W., Huber, R., Bacher, A., Rohdich, F. Structural basis of fosmidomycin action revealed by the complex with 2-C-methyl-D-erythritol 4-phosphate synthase (IspC). Implications for the catalytic mechanism and anti-malaria drug development. J. Biol. Chem., 2003, 278, 18401-18407.

Kuzuyama, T., Takahashi, S., Takagi, M., Seto, H. Characterization of 1-deoxy-D-xylulose 5-phosphate reductoisomerase, an enzyme involved in isopentenyl diphosphate biosynthesis and identification of its catalytic amino acid residues. J. Biol. Chem., 2000, 275, 19928-19932.

[44] Houry, W.A., Frishman, D., Eckerskorn, C., Lottspeich, F., Hartl, F.U. Identification of in vivo substrates of the chaperonin GroEL. Nature., 1999, 402, 147-154.

[45] Botha, M., Pesce, E.-R., Blatch, G.L. The Hsp40 proteins of Plasmodium falciparum and other apicomplexa: Regulating chaperone power in the parasite and the host. Int. J. Biochem. Cell Biol., 2007, 39, 1781-1803.

[46] Spork, S., Hiss, J.A., Mandel, K., Sommer, M., Kooij, T.W.A., Chu, T., Schneider, G., Maier, U.G., Przyborski, J.M. An unusual ERAD-like complex is targeted to the apicoplast of Plasmodium falciparum. Eukaryot. Cell., 2009, 8, 1134-1145.

[47] LaCount, D.J., Vignali, M., Chettier, R., Phansalkar, A., Bell, R., Hesselberth, J.R., Schoenfeld, L.W., Ota, I., Sahasrabudhe, S., Kurschner, C., Fields, S., Hughes, R.E. A protein interaction network of the malaria parasite Plasmodium falciparum. Nature. 2005, 438, 103-107.

[48] Misra, G., Ramachandran, R. Hsp70-1 from Plasmodium falciparum: Protein stability, domain analysis and chaperone activity R. Biophysical Chem., 2009, 142, 55-64.

[49] Sato, S., Wilson, R.J. The plastid of Plasmodium spp: A target for inhibitors. Curr, Top. Microbiol. Immunol., 2005, 295, 251-273.

[50] Gießmann, D., Heidler, P., Haemers, T., Van Calenbergh, S., Reichenberg, A., Jomaa, H., Weidemeyerd, C., Sanderbrand, S., Wiesner, J., Link, A. Towards new antimalarial drugs: synthesis of non-hydrolyzable phosphate mimics as feed for a predictive QSAR study on 1-Deoxy-d-xylulose-5-phosphate reductoisomerase inhibitors. Chem. Biol., 2008, 5, 643-656.

[51] Rohdich, F., Lauw, S., Kaiser, J., Feicht, R., Köhler, P., Bacher, A., Eisenreich, W. Isoprenoid biosynthesis in plants - 2C-methyl-derythritol-4-phosphate synthase (IspC protein) of Arabidopsis thaliana. FEBS J., 2006, 273, 4446-4458.
Grolle, S, Bringer-Meyer, S., Sahm, H. Isolation of the $d x r$ gene of Zymomonas mobilis and characterization of the 1-deoxy-Dxylulose 5-phosphate reductoisomerase. FEMS Microbiol. Lett., 2000, 191, 131-137.

DeLano, W.L. The PyMOL Molecular Graphics System, 2002, DeLano Scientific, San Carlos, CA, USA. http://www.pymol.org. 